This item was submitted to Loughborough's Research Repository by the author.

Items in Figshare are protected by copyright, with all rights reserved, unless otherwise indicated.

\title{
Influence of ion assisted deposition on interface broadening in Fe/Al multilayers investigated by medium energy ion scattering
}

PLEASE CITE THE PUBLISHED VERSION

http://dx.doi.org/10.1016/j.vacuum.2009.06.002

\section{PUBLISHER}

(C) Elsevier

\section{VERSION}

AM (Accepted Manuscript)

\section{LICENCE}

CC BY-NC-ND 4.0

\section{REPOSITORY RECORD}

Al-Busaidy, Mohamed S.K., P. Bailey, T.C.Q. Noakes, and Michael D. Cropper. 2019. "Influence of Ion Assisted Deposition on Interface Broadening in Fe/al Multilayers Investigated by Medium Energy lon Scattering". figshare. https://hdl.handle.net/2134/10741. 
This item was submitted to Loughborough's Institutional Repository (https://dspace.lboro.ac.uk/) by the author and is made available under the following Creative Commons Licence conditions.

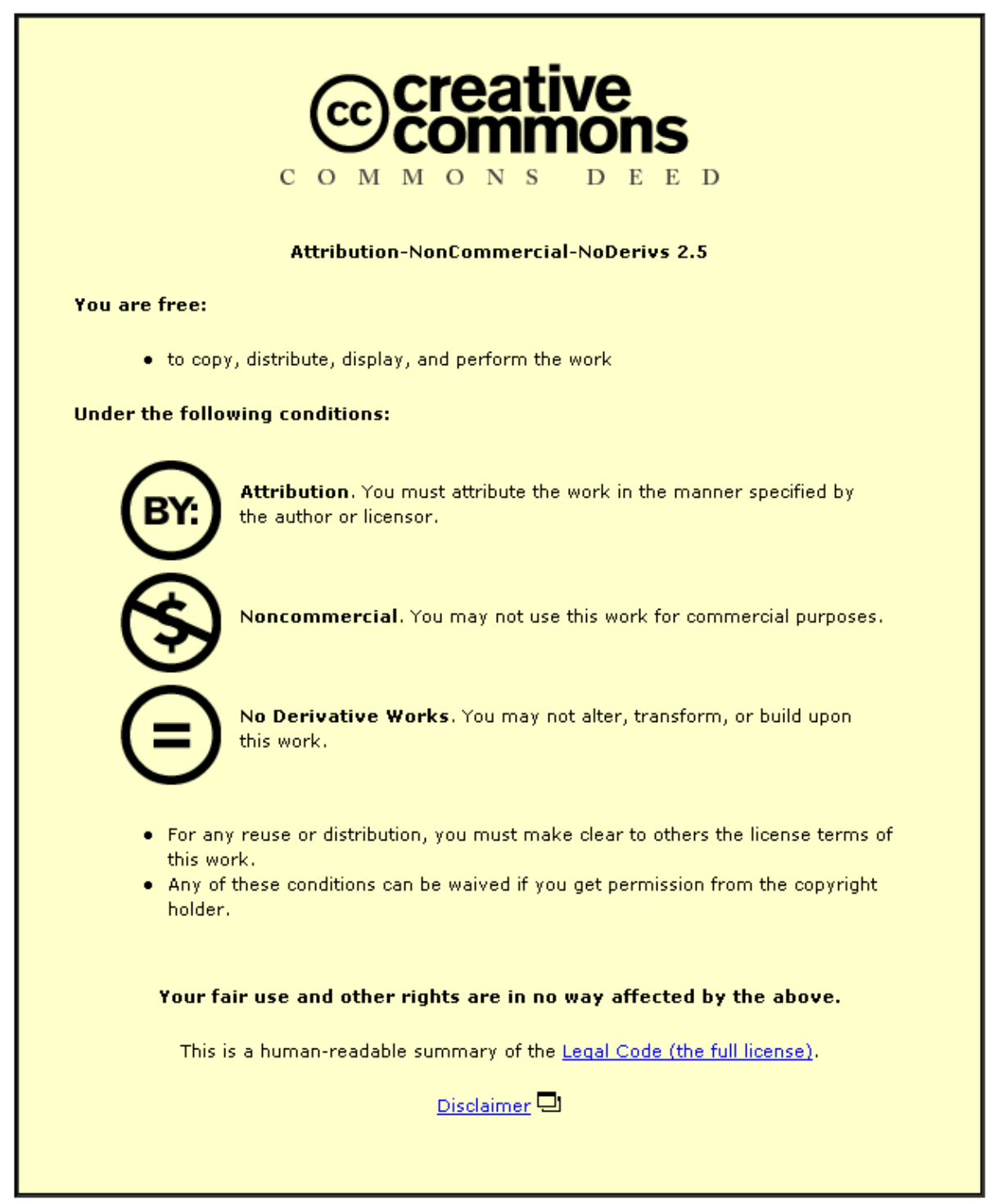

For the full text of this licence, please go to: http://creativecommons.org/licenses/by-nc-nd/2.5/ 


\title{
Influence of ion assisted deposition on interface broadening in FelAl multilayers investigated by medium energy ion
} scattering

M. S. Al-Busaidi ${ }^{1}$, P. Bailey ${ }^{3}$, T. C. Q. Noakes ${ }^{3}$, M. D. Cropper ${ }^{2,4}$

${ }^{1}$ College of Science, Physics Department, Sultan Qaboos University, P.O. Box 36, Al-Khod 123, Sultanate of Oman

${ }^{2}$ Department of Physics, Loughborough University, Loughborough, LE11 3TU, United Kingdom

${ }^{3}$ STFC, Daresbury Laboratory, Daresbury, Warrington, WA4 4AD, United Kingdom

${ }^{4}$ Main author email address: $\underline{\text { m.d.cropper@lboro.ac.uk }}$ Fax: 01509223986

Tel: 01509223308

\begin{abstract}
Trilayers of $\mathrm{Al} / \mathrm{Fe} / \mathrm{Al}$ and $\mathrm{Al} / \mathrm{Fe}$ multilayers produced by magnetron sputtering both with and without ion assistance have been depth profiled using Auger electron spectroscopy and medium energy ion scattering. Important differences are observed in the layer structure, with ion assisted deposition giving the narrowest $\mathrm{Al} / \mathrm{Fe}$ interfaces and so maintaining the most clearly defined layer structure. Both types of sputtering result in some oxygen contamination that modeling shows to be associated with the highly reactive Al layers.
\end{abstract}

Keywords: Iron; aluminium; multilayer; MEIS; magnetron; depth-profiling; trilayer. 


\section{Introduction}

Multilayers of Fe and Al have been the subject of several investigations motivated both by the interest in their soft magnetic properties, including low coercivity and high saturation magnetisation [1] and by their use as an example of solid state reaction [2]. Multilayers with bilayer periods in the range of 3-60 $\mathrm{nm}$ have been deposited by electron beam evaporation $[2,3,4]$, RF sputter deposition [1, 5], DC sputter deposition [6] and pulsed laser deposition [7]. Aside from the magnetic properties, the main focus has been on the degree of intermetallic compound formation at room temperature and following a post-deposition anneal. The consensus is that even at room temperature a broad interface forms by reaction between Fe and Al that is of the order of 1-2 nm wide $[3,7]$. Upon annealing to sufficiently high temperature, the film converts to a solid-state compound $[3,4]$. The motivation for the study reported in this paper is to discover if the use of ion assisted deposition, which results in the production of denser, smoother layers, can reduce the width of the interface between the Fe and Al layers.

Magnetron sputter deposition is one of the most common methods for the deposition of metallic multilayers [8]. Two important features of sputter deposition are the higher energy of arriving species compared with evaporation and the back reflection of Ar neutrals from the target onto the growing film [9]. These neutral atoms are produced by neutralisation of energetic ions in the cathode dark space and upon back-reflection from more massive target atoms impinge upon the growing film. The combined effect of 
these features tends to produce denser, smoother films. When sputter depositing a light element such as Al, however, there will be little backreflected flux of Ar neutrals as the mass of Ar exceeds that of Al. This is may be replaced by using a variant of ion-assisted deposition. Rather than using a separate ion source, an unbalanced magnetron can provide the flux necessary to smoothen and densify the film. In an unbalanced magnetron, the flux from the outer ring of magnets does not equal that from the inner ring causing flux to leak away to the substrate. This flux draws plasma to the growing film surface where it may be accelerated by applying a negative bias to the substrate. This impinging ion flux has a strong effect on the film microstructure and could suppress the development of roughness in multilayers and may also suppress interdiffusion by creating denser films.

A previous investigation of the influence of bias sputtering on Fe/Al multilayers used grazing incidence reflectivity primarily to determine the average roughness of the interfaces [10]. It was found that the use of bias sputtering reduced the roughness of the Fe-Al interfaces, reduced the overall roughness of the film and produced samples that gave much clearer Bragg peaks from the superlattice period. A comparison of the curves from bias and non-bias sputtered multilayers would indicate a much better bilayer structure in the bias sputtered case, but the reciprocal space nature of X-ray analysis makes the formation of a clear cut picture of the structures a non-trivial exercise. A simple depth profile through the films would give a clearer picture, but the narrowness of the layers makes this difficult by traditional means. Depth 
profiling by Auger electron spectroscopy (AES) and ion erosion is often limited in depth resolution to a few nm [11] by the electron escape depth and crater roughness. Whilst secondary ion mass spectrometry can do better than this, it is difficult to quantify.

Medium energy ion scattering spectroscopy (MEIS) $[12,13]$ is a variant of Rutherford backscattering spectroscopy (RBS) $[14,15]$ that gives greater surface sensitivity and greater depth resolution whilst retaining the quantifiable nature. It utilises typically $\mathrm{He}^{+}$or $\mathrm{H}^{+}$ions with energy of the order of $100 \mathrm{keV}$ rather than the MeV energies found in RBS. MEIS can give elemental analysis of surfaces and thin films with sub-nanometre resolution $[13,16,17,18,19,20]$.

In this paper we report a study on the use of MEIS to analyse Al/Fe multilayers and trilayers that have been produced by sputtering from an unbalanced magnetron both with and without substrate bias. We find that structures deposited without plasma assistance show a broad interface between the Fe and Al layers, causing the layer-by-layer structure to largely break down at the top of a multilayer stack. The use of bias sputtering is shown to reduce the interface width and to help maintain the multilayer integrity. In addition, the utility of MEIS for the surface analysis of these nanometre scale structures (see also reference [21]) is further demonstrated.

\section{Experimental}


Two types of samples were deposited: Al/Fe/Al trilayers and Fe/Al multilayers. In each case, they were deposited onto Si substrates with a native oxide both without ion assistance by grounding the substrate and with ion assistance by biasing it at $-200 \mathrm{~V}$. The deposition was carried out utilising d.c. planar magnetron sputtering in an $\mathrm{Ar}^{+}$atmosphere (99.995\% pure source gas) in a modified Nordiko system that has been described elsewhere $[22,23]$. The samples were transferred outside of vacuum to separate systems for AES and MEIS analysis as detailed below.

The trilayers and multilayers were deposited sequentially onto Si(111) wafers of $0.525 \mathrm{~mm}$ thickness that had been cleaved to squares of size of $2 \mathrm{~cm} \times 2$ $\mathrm{cm}$. Al and Fe targets of $5 \mathrm{~cm}$ diameter were used, the Al target being $99.95 \%$ pure and $2.0 \mathrm{~mm}$ thick and the Fe target being $99.95 \%$ pure and only 0.25 $\mathrm{mm}$ thick (to reduce magnetic shorting of the magnetron). The Al target was mounted into an unbalanced magnetron so that some of the argon plasma could leak away and impinge upon the substrate during growth. The Fe target was mounted into a balanced magnetron as Fe is sufficiently heavy to backreflect Ar neutral species onto the growing film. The base pressure of the deposition system was $1 \times 10^{-7}$ mbar and a sputtering gas pressure of $2.6 \times 10^{-3}$ mbar was used with a target power of $70 \mathrm{~W}$. This gave approximate deposition rates of $0.33 \mathrm{~nm} \mathrm{~s}^{-1}$ for $\mathrm{Al}$ and $0.15 \mathrm{~nm} \mathrm{~s}^{-1}$ for Fe, which is comparable with those used in other reports $[2,4]$. The target to substrate distance was $10 \mathrm{~cm}$ with the two magnetrons angled towards the centre of the substrate. The Al and Fe layers were alternately sputtered onto the substrate 
using time-controlled, pneumatically actuated shutters on the magnetron sources. The deposition rates had previously been calibrated using X-ray reflectivity measurements and nominal structures that were deposited were $\mathrm{Si}(111) / \mathrm{SiO}_{x(\text { native) }} / \mathrm{Al}(4 \mathrm{~nm}) / \mathrm{Fe}(3.7 \mathrm{~nm}) / \mathrm{Al}(4.0 \mathrm{~nm})$ for the trilayers and $\mathrm{Si}(111) / \mathrm{SiO}_{x(\text { native })} /[\mathrm{Fe}(3.7 \mathrm{~nm}) / \mathrm{Al}(4.0 \mathrm{~nm})]_{19} / \mathrm{Fe}(1 \mathrm{~nm})$ for the multilayers .

The multilayer films were transferred under normal atmosphere conditions for analysis by AES using a JEOL Jamp 7100 system. The samples were analysed using a primary electron energy of $3 \mathrm{keV}$, a current of $0.7 \mu \mathrm{A}$ and a spot-size of $100 \mu \mathrm{m}$ diameter. The transitions used for analysis were the Fe $703 \mathrm{eV}$ (LMM), Al $68 \mathrm{eV}$ (LVV) and Si $92 \mathrm{eV}$ (LVV). Depth profiling was carried out using $\mathrm{Ar}^{+}$bombardment.

The trilayer and multilayer samples were transferred under normal atmosphere conditions to the UK National MEIS Facility at Daresbury Laboratory [24]. This facility has an ultra-high vacuum end station with a loadlock for sample introduction. $\mathrm{He}^{+}$ions with a nominal energy of $100 \mathrm{keV}$ were directed at the target at an incidence angle of $45^{\circ}$ to the surface normal and were detected at a scattering angle of $90^{\circ}$.

\section{Results and Discussion}

From the AES analysis, it was found that the main contaminants were a surface $\mathrm{C}$ peak, some surface $\mathrm{O}$ and $\mathrm{O}$ distributed throughout the multilayer at a level of about $10 \%$ atomic fraction. The thickness of the surface oxide 
was about $8 \mathrm{~nm}$ (which would encompass much of the first bilayer). The presence of the bulk oxide is not unexpected for the deposition of Al by sputtering in high vacuum, and has been reported by other authors [1]. It is difficult to deposit pure Al films by this method due to its reactivity, but since the main interest in Fe/Al films is in the magnetic properties of the Fe layers [25] some contamination of the Al layers is tolerable.

The Auger depth profile of the two multilayer samples is shown in Figure 1. The near surface region (small etch time) is shown with an expanded scale as in this region there is reasonable depth resolution. With increasing etch time the resolution degrades due to statistical effects in the sputtering. From the graph it can be seen that the surface is oxidised to a greater extent as would be expected. The first Fe layer can be identified in the multilayer grown with bias, but not in the one grown without bias.

The results of the MEIS analyses of the trilayer and multilayer samples are shown in Figs 2 and 3 respectively. When viewing these spectra it is important to bear in mind the mechanism of MEIS depth profiling. The scattering event is a binary collision between the $\mathrm{He}^{+}$ion and the nucleus of the target atom. In this collision the target atom recoils so reducing the energy of the scattering $\mathrm{He}^{+}$ion; the lighter the target atom, the greater this recoil energy loss of the scattered ion. Thus in Fig. $2 \mathrm{He}^{+}$ions scattered from the surface Al layer produces a peak in the spectrum at around $73 \mathrm{keV}$, but the peak due to scattering from the more massive Fe layer is at a higher energy. There is a second mechanism of energy loss in the scattered $\mathrm{He}^{+}$ions that is due 
quasi-continuous inelastic losses to the electron sea that the ions pass through in travelling to and from the target atom. Thus the energy loss of $\mathrm{He}^{+}$ ions scattering from the deeper Al layer in the trilayers (Fig. 2) is greater and these ions give signal around $65 \mathrm{keV}$.

To assist with a more quantitative interpretation of the MEIS spectra, simulations of the spectra were made using a macro that runs in Igor Pro [26]. The simulation accounts for the Z-dependent Rutherford scattering crosssection, the stopping power of the elements calculated using SRIM [27], a composition dependent stopping power, loss of resolution with depth due to straggling [28] and the path length defined by the analysis geometry. A correction is made to the pure Rutherford cross-section to compensate for core screening [29]. Since the $\mathrm{Al}$ is highly oxidised, a stopping power for $\mathrm{Al}_{2} \mathrm{O}_{3}$ was calculated (at a density of $3100 \mathrm{kgm}^{-3}$ which is typical for thin films of alumina). The exact stoichiometry of the alumina films will not greatly affect the stopping power since the stopping powers of pure $\mathrm{Al}$ and $\mathrm{Al}_{2} \mathrm{O}_{3}$ differ by only $\sim 10 \%$. The results of the simulations are shown as solid lines superimposed on the spectra in Figs. 2 and 3 and the compositional model used to generate them is shown in the figure insets.

Looking first at the spectrum from an Al/Fe/Al trilayer deposited using a grounded substrate shown in Fig. 2(a) several features are apparent. The scattering from the surface Al layer can be seen between 70 and $73 \mathrm{keV}$, but the scattering from the subsurface Al layer is poorly resolved from the continuum due to the Si substrate (below $65 \mathrm{keV}$ ) as they have similar 
masses. The peak due to scattering from the central Fe layer is clearly resolved between 80 and $84 \mathrm{keV}$. The Fe signal has some notable features: the counts at a higher energy than the peak are non-zero indicating broadening of the interface with the surface Al layer, additionally there is a shoulder between 76 and $80 \mathrm{keV}$ that represents Fe that has broadening of the interface with the second Al layer. The O contamination of the film is also clear: the surface peak of $\mathrm{O}$ is apparent at $58 \mathrm{keV}$, below which there is a gap to another $\mathrm{O}$ peak at $54 \mathrm{keV}$. The model indicates that the $\mathrm{O}$ is mainly in two layers, each associated with one of the Al layers. The broadening of the interface between the Fe and Al layers may be due to either interdiffusion or roughening of the interfaces. As the MEIS signal is integrated over a comparatively large area, broadening of interfaces by either mechanism cannot be distinguished. It is know from X-ray reflectivity measurements that bias sputtering reduces the apparent roughness of interfaces, and that mechanism will be active here. Looking at the inset to Figure 2(a) it can be seen that to fit the data well it is necessary to broaden the Fe layer all the way through the lower Al layer, indicating that intermixing may be contributing to the broadening.

The spectrum from the trilayer deposited with ion assistance using a substrate bias of $-200 \mathrm{~V}$ is shown in Fig. 2(b). This has several differences from the trilayer deposited without bias. Firstly the peak corresponding to the Al surface layer is narrower (at $2.6 \mathrm{~nm}$ cf. 4.4 for the non ion assist) indicating that the net deposition rate in $\mathrm{nm} \mathrm{s}^{-1}$ is lower with the bias sputter. The total 
amount of Al present (indicated by the peak integral in MEIS) is reduced, probably due to a re-sputtering of loosely bound material. The low energy shoulder corresponding to the broadening of the Fe interface with the lower Al layer is suppressed. Again $\mathrm{O}$ is present mainly in two layers. It should be pointed out that, although the $O$ signal has been included in both the models, the signal to noise levels of the O peaks on top of the Si signal is such that we do not necessarily get an accurate $\mathrm{O}$ to Si ratio. Nevertheless, in both cases, the $\mathrm{O}$ appears to be associated with the Al rather than the Fe. Since the samples were transported in air it is to be expected that the surface Al layer will be oxidised. For the buried Al layer however, the positions calculated in the model for the back edge of the buried Al layer, the peaks in the O signal and the front edge of the Si suggest that a graded interface of $\mathrm{Al} / \mathrm{Si} / \mathrm{O}$ has formed.

Fig. 3 shows the spectra of Fe/Al multilayers and, unlike Fig. 2, the signal here is due mainly to Fe. The MEIS profile of the top two Fe layers for the multilayer deposited without bias sputtering is shown offset in Fig. 3. Only two layers were probed since any oscillations in the data at lower energies will become less intense due to straggling. The two Fe layers are poorly defined, with little change in ion scattering counts between the centre of the two layers at about $82.5 \mathrm{keV}$ and about $77 \mathrm{keV}$ and the notional absence of Fe due to the presence of the Al layer at around $79.7 \mathrm{keV}$. The lack of contrast may be due to interdiffusion or, as MEIS depth profiles must be referenced to the top surface, to integrated roughness within the film stack. For whatever reason, 
during the growth of the nineteen bilayer stack, the layer-by-layer structure has almost completely broken down.

By contrast the bias sputtered multilayer (using a substrate bias of $-200 \mathrm{~V}$ ) has clearly resolved Fe layers which can be seen at scattering energies of around 82 and $76 \mathrm{keV}$ with a dip due to the Al layer between them. In addition, the narrow Fe capping layer, although weak, is clearly resolved whereas in the non-bias deposited sample it is visible but not well-resolved. The model shown superimposed on the bias sputtered spectrum is the sum of all the Gaussian peaks shown. These are separated by a fixed energy of 4.9 $\mathrm{keV}$ and, to model straggling, broaden by a fixed amount as the peaks decrease in energy. The model is therefore based on an ideal multilayer structure with a $7.2 \mathrm{~nm}$ repeat distance. Any Al signal which should begin to appear at $\sim 73 \mathrm{keV}$ cannot be resolved in the data, presumably due to its much lower cross section. As in the case of the trilayers there is some broadening of the interface between the Fe and the first Al layer, and between the first layer Fe and the second Al layer which is not seen in deeper layers and clearly the fourth Al layer is slightly thinner than the shallower ones. Hence the model indicates that the layer structure is very close to ideal. However, in order to get a good match to the data the model needs to include a sloping background, which is difficult to explain. Possibly this is related to the unresolved Al signal that the Fe signal overlies or due to the increased scattering cross-section with reducing ion energy. This latter effect is know in RBS but is not always apparent in MEIS. Nonetheless, it is clear that bias 
sputtering assists the maintenance of the layer-by-layer structure throughout the four bilayers investigated.

\section{Conclusion}

It has been demonstrated that trilayers and multilayers of Fe and Al deposited by dc magnetron sputtering show broadened interfaces between the Fe and Al layers. The application of bias sputtering to give ion assistance to the growth helps reduce this broadening and helps maintain the integrity of the layers. Both types of sputtering result in some oxygen contamination that is associated with the highly reactive Al layers. The importance of using surface analysis methods such as MEIS in the investigation of such systems has been demonstrated.

\section{Acknowledgements}

The EPSRC provided access to the UK National MEIS Facility (Grant reference EP/E003370/1).

\section{References}

[1] D'Orazio F, Gubbiotti G, Lucari F, Tassoni E. Journal of Magnetism and Magnetic Materials 2002;242-245:535-537

[2] Mengucci P, Majni G, Di Cristoforo A, Checchetto R, Miotello A, Tosello C, Principi G. Thin Solid Films 2003;433;205-210

[3] Brajpuriya R, Sharma A, Tripathi S, R. Reddy VR, Chaudhari SM. Journal of Physics: Condensed Matter, 2006;18:1197-1210 
[4] Checchetto R, Tosello C, Miotello A, Principi G. Journal of Physics: Condensed Matter, 2001;13:811-821

[5] Bhattacharya P, Ishihara KN, Chattopadhyay K. Materials Science and Engineering A, 2001;304-306:250-254

[6] Haeiwa T, Negoro H, Matsumoto M. Journal of Applied Physics, 1991;69:5346-5348

[7] Meyer DC, Gawlitza P, Richter K, Paufler P. Journal of Physics D: Applied Physics, 1999;32:3135-3139

[8] Fullerton EE, Kelly DM, Guimpel J, Schuller IK, Bruynseraede Y. Physical Review Letters, 1992;68:859-862

[9] Maissel LI and Glang R. Handbook of thin film technology. New York: McGraw-Hill,1970. pp 4.1- 4.44

[10] Al-Busaidy MS, Crapper MD. Journal of Vacuum Science and Technology A, 2006;24:595-599

[11] Sykes DE, Hall DD, Thurstans RE, Walls JM. Applications of Surface Science, 1980;5:103-106

[12] van der Veen JF. Surface Science Reports, 1985;5:199-287

[13] Tromp RM, in: Practical Surface Analysis Volume 2 Ion and Neutral Spectroscopy, second edition, edited Briggs D. Chichester: Wiley 1992.

[14] Taguchi T, Yokogawa T. Journal of Physics D: Applied Physics, 1984;17:1067-1082

[15] Kashani H. Thin Solid Films, 1996;288:50-56

[16] Gusev EP, Lu HC, Gustafsson T, Garfunkel E. Physical Review B 1995;52:1759-1775

[17] Copel M, Cartier E, Gusev EP, Guha S, Bojarczuk N, Poppeller M. Applied Physics Letters ,2001;78:2670-2672

[18] Werner M, van den Berg JA, Armour DG, Vandervost W, Collart EHJ, Goldberg RD, Bailey P, Noakes TCQ. Nuclear Instruments and Methods in Physics Research B, 2004;216:67-74

[19] Giubertoni D, Bersani M, Barozzo M, Pederzoli S, lacob E, van den Berg JA, Werner M. Applied Surface Science, 2006;252:7214-7217

[20] Kim YP, Choi SK, Kim HK, Moon DW. Applied Physics Letters, 1997;71:3504-3506

[21] Noakes TCQ, Bailey P, Hucknall PK, Donovan K, Howson MA. Physical Review B, 1998;58:4934-4941

[22] Telling ND, Petty M, Crapper MD. Journal of Vacuum Science and Technology A, 1998;16:145-147 
[23] Telling ND, Guilfoyle SJ, Lovett DR, Tang CC, Crapper MD, Petty M. Journal of Physics D: Applied Physics, 1998;31:472-481

[24] Bailey P, Noakes TCQ, Woodruff DP. Surface Science, 1999;426:358372

[25] Chérif SM, Bouziane K, Roussigné $Y$, Al-Busaidy M. Materials Science and Engineering B, 2007;138:16-21

[26] Igor Pro, Wavemetrics. (http://www.wavemetrics.com/)

[27] Ziegler JF. SRIM Code, Yorktown Heights, NY. (http://www.SRIM.org)

[28] Okazawa T, Shibuya K, Nishimura T, Kido Y. Nuclear Instruments and Methods in Physics Research Section B: Beam Interactions with Materials and Atoms, 2007;256:1-5

[29] Andersen HH, Besenbacher F, Loftager P, Moller W. Physical Review A, 1980;21:1891-1901 


\section{Figure Captions}

Figure 1: Auger electron spectroscopy depth profile of multilayer samples grown (a) without a substrate bias and (b) with a substrate bias. Note the break point in the etch time axis to show an expanded depth scale near the surface.

Figure 2: Medium energy ion scattering from Al/Fe/Al trilayers deposited onto Si using (a) $0 \mathrm{~V}$ bias and (b) $-200 \mathrm{~V}$ bias. Superimposed on the data are simulations (solid line) based on the atomic concentrations with depth shown in the insets. On Fig. 2a indications are given for the approximate regions corresponding to the four elemental constituents.

Figure 3: Medium energy ion scattering from Fe/Al multilayers deposited onto Si using $0 \mathrm{~V}$ bias and $-200 \mathrm{~V}$ bias ion assist. Superimposed on the data are a simulation (solid line). For clarity, the $0 \mathrm{~V}$ bias spectrum has been moved upwards along the y-axis. 
Figure 1

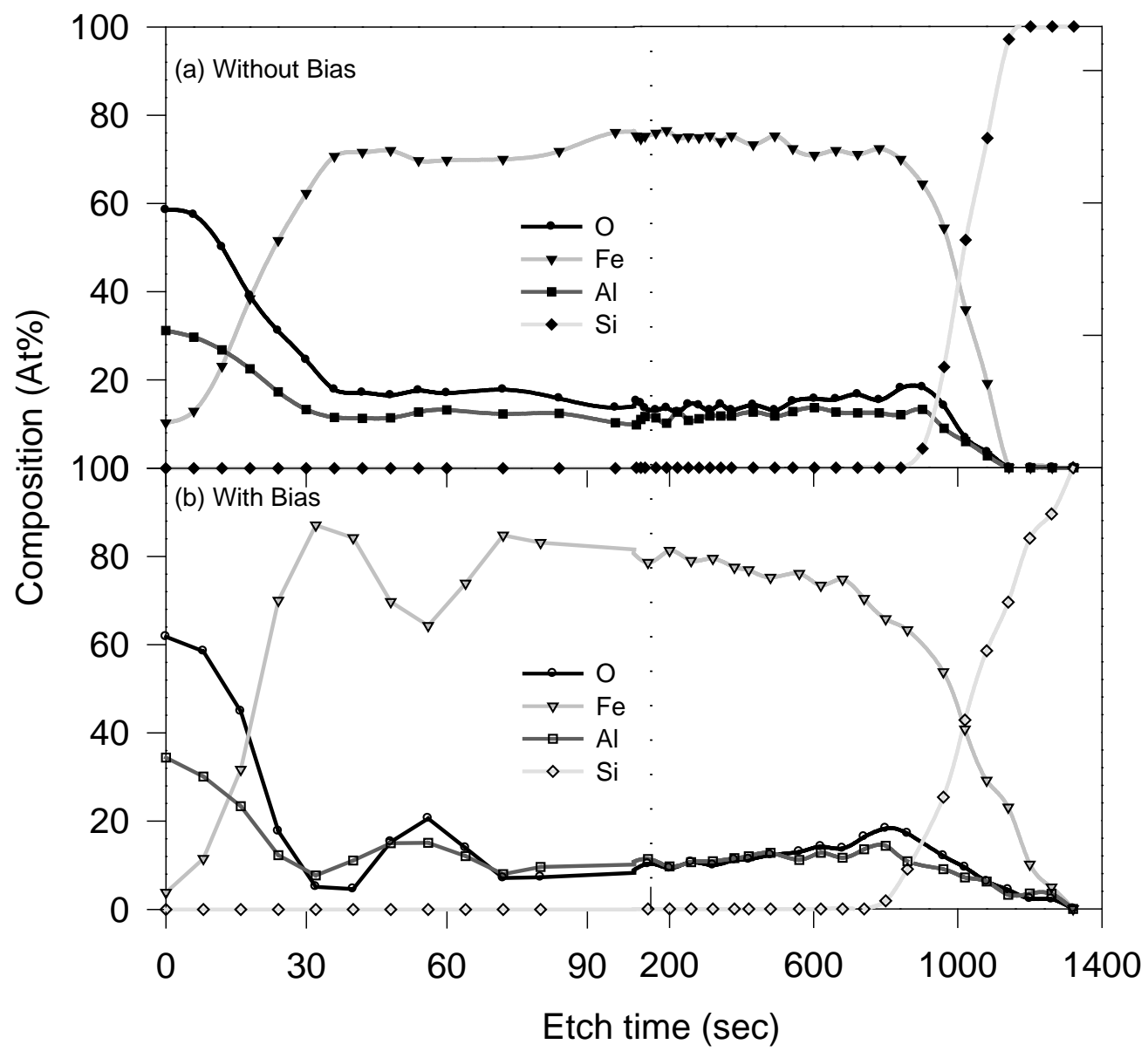


Figure $2 \mathrm{a}$

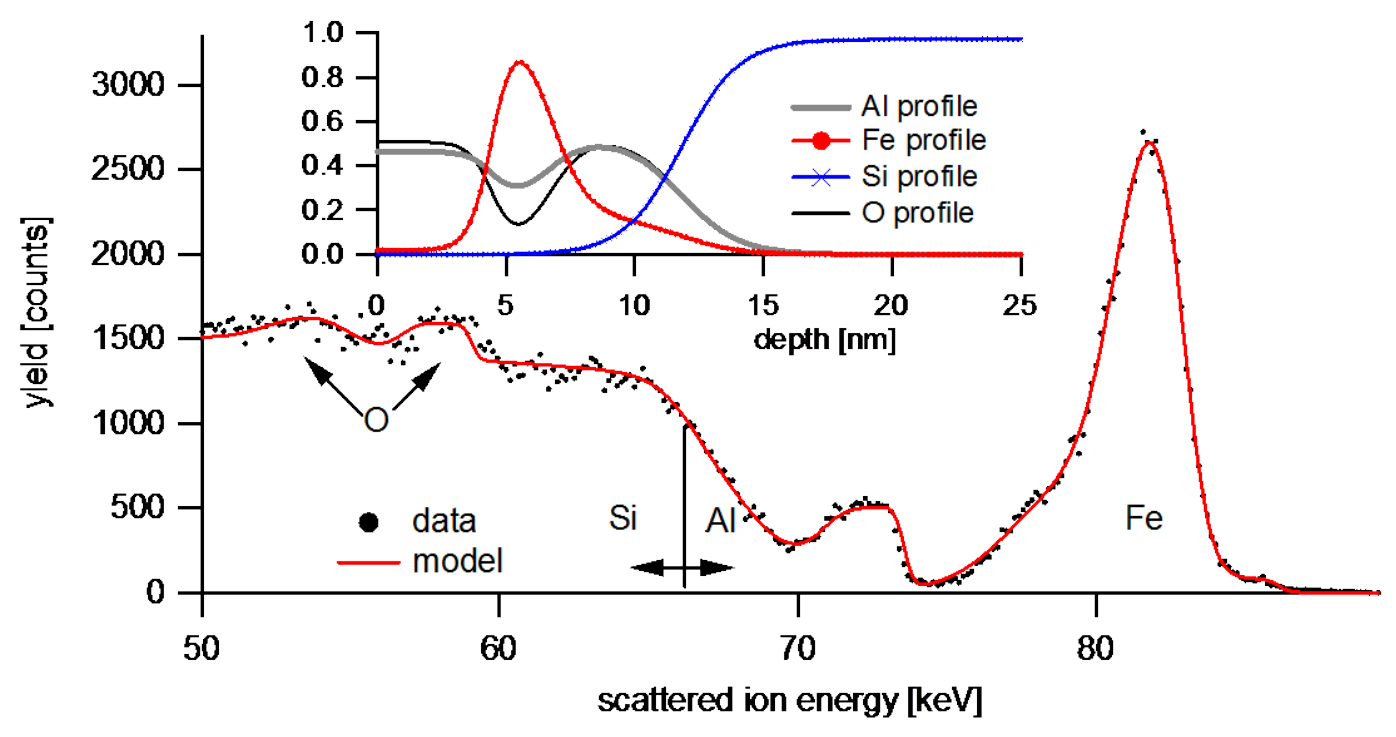

Figure 2b

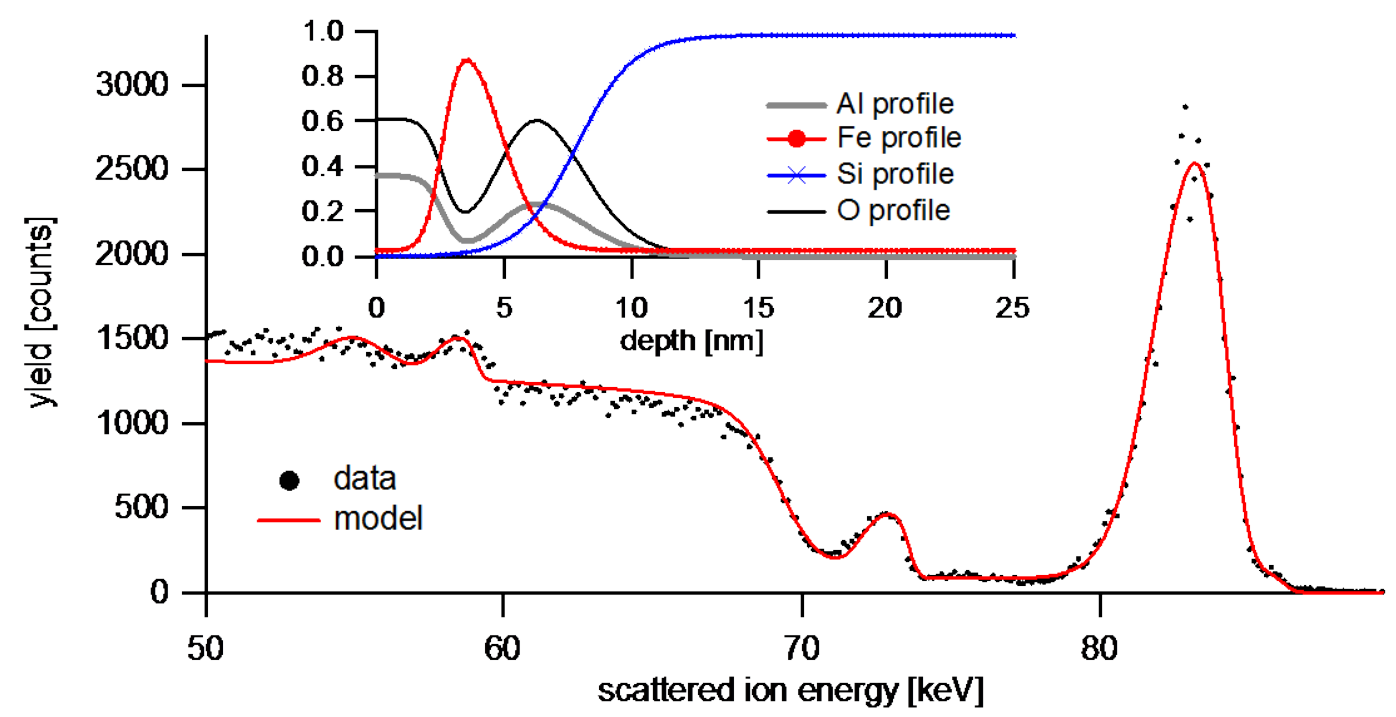


Figure 3

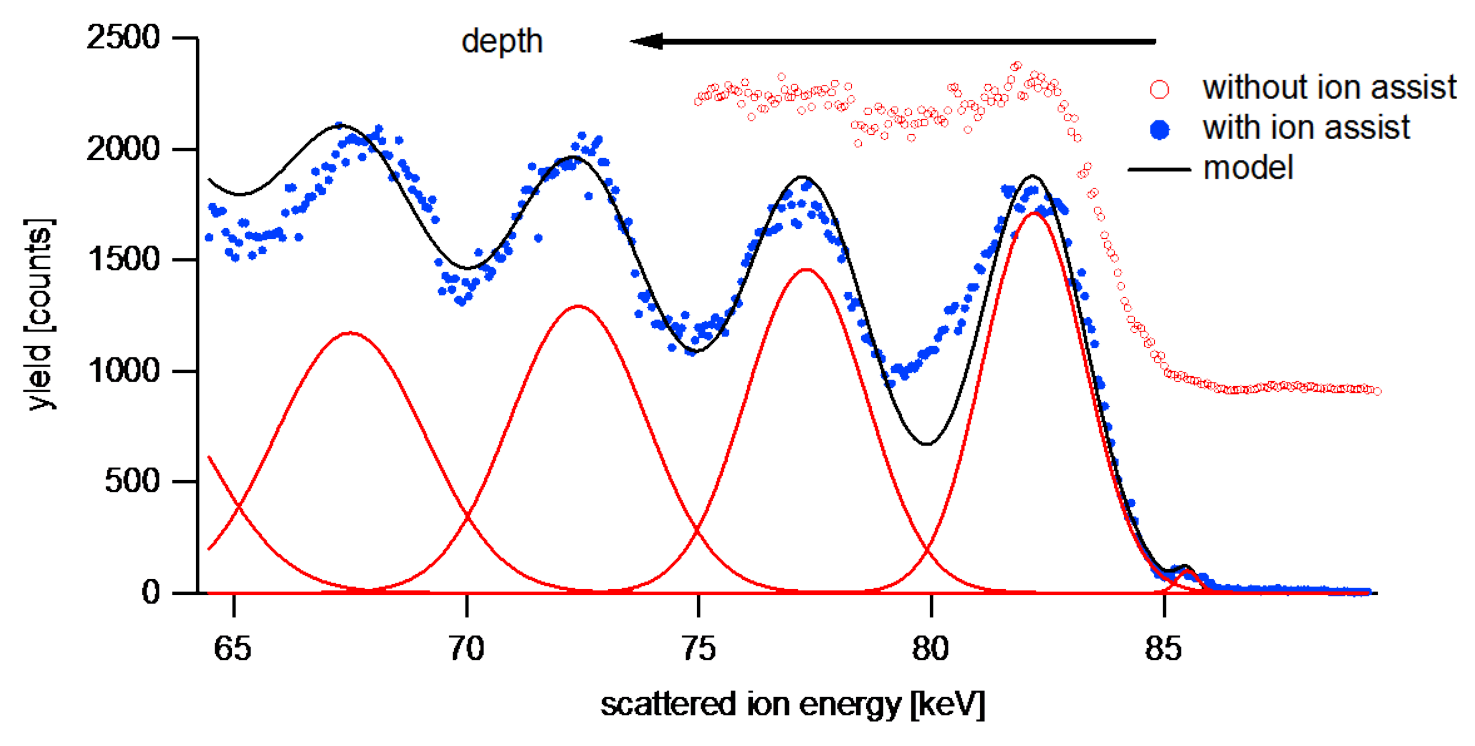

